Appl. Set-Valued Anal. Optim. 3 (2021), No. 1, pp. 55-73

Available online at http://asvao.biemdas.com

https://doi.org/10.23952/asvao.3.2021.1.06

\title{
CONVERGENCE THEOREMS OF COMMON SOLUTIONS OF VARIATIONAL INEQUALITY AND $f$-FIXED POINT PROBLEMS IN BANACH SPACES
}

\author{
GETAHUN BEKELE WEGA, HABTU ZEGEYE* \\ Department of Mathematics and Statistical Sciences, \\ Botswana International University of Science and Technology, Palapye, Botswana
}

\begin{abstract}
The purpose of this paper is to introduce the concept of a Bregman relatively $f$-nonexpansive mappings and study common solutions of the variational inequality problem involving Lipschitz monotone mappings and the fixed point problem of Bregman relatively $f$-nonexpansive mappings. Under some mild conditions, we obtained strong convergence theorems in real reflexive Banach spaces.

Keywords. Bregman relatively $f$-nonexpansive; $f$-fixed point; Monotone mapping; Variational inequality; Zero point.
\end{abstract}

\section{INTRODUCTION}

Let $E$ be a real Banach space with its dual $E^{*}$. Let $C$ be a nonempty subset of $E$. A mapping $A: C \rightarrow E^{*}$ is said to be monotone if

$$
\langle A x-A y, x-y\rangle \geq 0, \quad \forall x, y \in C .
$$

$A: C \rightarrow E^{*}$ is said to be maximal monotone if its graph $G p h(A), G p h(A)=\left\{(x, A x) \in E \times E^{*}\right.$ : $x \in C\}$, is not properly contained in the graph of any other monotone mapping. $A: C \rightarrow E^{*}$ is said to be $\alpha$-inverse strongly monotone if there exists a positive real number $\alpha$ such that

$$
\langle A x-A y, x-y\rangle \geq \alpha|| A x-A y \|^{2}, \quad \forall x, y \in C .
$$

It is known that $\alpha$-inverse strongly monotone mappings are monotone and Lipschitz continuous. Let $T: C \rightarrow E$ be a mapping. The set of fixed points of $T$ is defined by $F(T):=\{x \in C: T x=x\}$ in this paper. Recall that $T$ is said to be contractive and if there exists a constant $L \in(0,1)$ such that $\|T x-T y\| \leq L\|x-y\|, \forall x, y \in C$. Furthermore, $T$ is said to be nonexpansive if $L=1$, that is, $\|T x-T y\| \leq\|x-y\|, \forall x, y \in C$. $T$ is said to be quasi-nonexpansive if $F(T) \neq \emptyset$ and $\|T x-T p\| \leq\|x-p\|$ for all $x \in C$ and $p \in F(T)$. Recall that the mapping $I-T$, where $I$ denotes the identity mapping, is said to be demiclosed at 0 if for any sequence $\left\{x_{n}\right\}$ in $C$ converging weakly to $x$ and $\left\|(I-T) x_{n}\right\| \rightarrow 0$, then $(I-T) x=0$.

Let $C$ be nonempty, closed and convex subset of space $E$ and let $A$ be a monotone mapping. The variational inequality problem (VIP) is formulated as finding a point

$$
x^{*} \in C \text { such that }\left\langle A x^{*}, x-x^{*}\right\rangle \geq 0, \quad \forall x \in C .
$$

${ }^{*}$ Corresponding author.

E-mail addresses: getahunbekele2012@gmail.com (G.B. Wega), habtuzh@yahoo.com (H. Zegeye).

Received May 26, 2020; Accepted June 30, 2020.

(C)2021 Applied Set-Valued Analysis and Optimization 
We denote the solution set of $(1.1)$ by $V I(C, A)$. This problem was first introduced by Hartman and Stampacchia [1] in 1966 and has been studied by several authors; see, e.g., [2, 3, 4, 5, 6, 7, 8] and there references therein.

In 2006, Nadezhkina and Takahashi [2] studied the following algorithm in a finite dimension Hilbert space setting. For any $x_{0} \in C,\left\{x_{n}\right\}$ is defined by

$$
\left\{\begin{array}{l}
y_{n}=P_{C}\left[x_{n}-\gamma_{n} A x_{n}\right] \\
x_{n+1}=\alpha_{n} x_{n}+\left(1-\alpha_{n}\right) P_{C}\left[x_{n}-\gamma_{n} A y_{n}\right], n \geq 0
\end{array}\right.
$$

where $A$ is $L$-Lipschitz continuous monotone mapping and $P_{C}$ is the metric projection from $H$ onto $C$. They proved that the sequence generated by (1.2) converges weakly to a solution of Problem (1.1) under certain conditions imposed on the control sequences $\left\{\gamma_{n}\right\}$ and $\left\{\alpha_{n}\right\}$.

In 2017, Thong and Hieu [3] introduced the following algorithm in a Hilbert space setting. For arbitrary $x_{0} \in H,\left\{x_{n}\right\}$ is defined by

$$
\left\{\begin{array}{l}
y_{n}=P_{C}\left[x_{n}-\gamma_{n} A x_{n}\right] \\
z_{n}=y_{n}-\gamma_{n}\left(A y_{n}-A x_{n}\right) \\
x_{n+1}=\alpha_{n} f\left(x_{n}\right)+\left(1-\alpha_{n}\right) z_{n}, n \geq 0
\end{array}\right.
$$

where $A$ is $L$-Lipschitz continuous on $H$ and $f: H \rightarrow H$ is a contractive mapping. They proved that the sequence generated by (1.3) converges strongly to $x^{*}=P_{V I(C, A)} f\left(x^{*}\right)$ provided that the control sequences $\left\{\gamma_{n}\right\}$ and $\left\{\alpha_{n}\right\}$ satisfy appropriate conditions.

Recently, several authors studied various algorithms for finding a common element of the set of fixed points of nonexpansive mapping and the set of solutions of variational inequality problems with Lipschitz monotone mappings; see, e.g., $[2,4,9,10,11]$ and the references therein.

In 2003, Takahashi and Toyoda [9] introduced the following scheme for finding a point in $V I(C, A) \cap F(T)$ in a Hilbert space setting. For arbitrary $x_{0} \in C,\left\{x_{n}\right\}$ is defined by

$$
x_{n+1}=\alpha_{n} x_{n}+\left(1-\alpha_{n}\right) T P_{C}\left(x_{n}-\gamma_{n} A x_{n}\right), n \geq 0,
$$

where $A: C \rightarrow H$ is $\alpha$-strongly monotone mapping, $T: C \rightarrow H$ is nonexpansive, $\left\{\alpha_{n}\right\}$ is a sequence in $(0,1)$ and $\left\{\gamma_{n}\right\}$ is a sequence in $(0,2 \alpha)$. They proved that if $V I(C, A) \cap F(T)$ nonempty, then the sequence generated by (1.4) converges weakly to some $x^{*} \in V I(C, A) \cap$ $F(T)$.

In 2005, Iiduka and Takahashi [4] investigated the following algorithm. For arbitrary $x_{0}, x \in$ $C,\left\{x_{n}\right\}$ is defined by

$$
x_{n+1}=\alpha_{n} x+\left(1-\alpha_{n}\right) T P_{C}\left(x_{n}-\gamma_{n} A x_{n}\right), n \geq 0,
$$

where $A: C \rightarrow H$ is an $\alpha$-strongly monotone mapping and $T: C \rightarrow H$ is a nonexpansive mapping. They proved that the sequence generated by (1.5) converges strongly to $x^{*}=P_{V I(C, A) \cap F(T)} x$ provided that the control sequences $\left\{\gamma_{n}\right\}$ and $\left\{\alpha_{n}\right\}$ satisfy appropriate conditions.

Recently, Zhang and Yuan [12] investigated an iterative process for approximating a common point of fixed points of nonexpansive mapping and solutions of the variational inequality problems with a finite family of $\alpha$-inverse strongly monotone mappings in the setting of Hilbert 
spaces. For arbitrary $x_{1}, \in C,\left\{x_{n}\right\}$ is defined by

$$
\left\{\begin{array}{l}
y_{n, i}=P_{C}\left[x_{n}-\gamma_{i} A x_{n}\right] \\
x_{n+1}=\alpha_{n} f\left(x_{n}\right)+\beta_{n} x_{n}+\gamma_{n} T \sum_{i=1}^{n} \eta_{i} y_{n, i}, n \geq 1
\end{array}\right.
$$

where $\left\|y_{n, i}-P_{C}\left(x_{n}-\gamma_{n} A x_{n}\right)\right\| \leq e_{n, i}$, and $\lim _{n \rightarrow \infty} e_{n, i}=0,\left\{\alpha_{n}\right\},\left\{\beta_{n}\right\}$ and $\left\{\gamma_{n}\right\}$ are real sequences in $(0,1)$ such that $\alpha_{n}+\beta_{n}+\gamma_{n}=\sum_{i=1}^{n} \eta_{i}=1,\left\{\gamma_{i}\right\}$ is a real sequence in $\left(0,2 \mu_{i}\right)$, $f: C \rightarrow C$ is fixed $\alpha$-contractive mapping, $T: C \rightarrow C$ is nonexpansive and $A_{i}: C \rightarrow H$ is $\mu_{i^{-}}$ inverse strongly monotone mapping for $1 \leq i \leq n$. They proved that the sequence generated by (1.6) converges strongly to $x^{*} \in \cap_{i=1}^{n}\left(V I, A_{i}\right) \cap F(T)$ provided that the control sequences satisfying certain conditions.

Let $E$ be a real Banach space with its dual $E^{*}$. For every $1<p<\infty$, the generalized duality mapping $J_{p}: E \rightarrow 2^{E^{*}}$ is defined by

$$
J_{p}(x)=\left\{y^{*} \in E^{*}:\left\langle x, y^{*}\right\rangle=\|x\|^{p},\left\|y^{*}\right\|=\|x\|^{p-1}\right\},
$$

for all $x \in E$. If $J=J_{2}$, then $J_{2}$ is called the normalized duality mapping. If $E=H$, a real Hilbert space, then $J=I$, where $I$ is the identity mapping on $E$.

Beyond Hilbert spaces, Tufa and Zegeye [13] investigated an algorithm for finding a common point of the set of fixed points of a relatively nonexpansive mapping $T: C \rightarrow E$ and the set of solutions of variational inequality problems with Lipschitz monotone mappings $A_{1}, A_{2}: C \rightarrow E^{*}$ in 2-uniformly convex and uniformly smooth real Banach spaces. They proved that the sequence generated by the following algorithm

$$
\left\{\begin{array}{l}
z_{n}=\Pi_{C} J^{-1}\left[J x_{n}-\gamma_{n} A_{2} x_{n}\right] \\
y_{n}=\Pi_{C} J^{-1}\left[J x_{n}-\gamma_{n} A_{1} x_{n}\right] \\
w_{n}=a_{n} J x_{n}+b_{n} J T x_{n}+c_{n} J\left(u_{n}\right)+d_{n} J\left(v_{n}\right) \\
x_{n+1}=\Pi_{C} J^{-1}\left[\alpha_{n} J u+\left(1-\alpha_{n}\right) w_{n}\right], n \geq 0
\end{array}\right.
$$

where $J$ is the normalized duality mapping, $u_{n}=\Pi_{C} J^{-1}\left[J x_{n}-\gamma_{n} A_{1} y_{n}\right], v_{n}=\Pi_{C} J^{-1}\left[J x_{n}-\right.$ $\left.\gamma_{n} A_{2} z_{n}\right],\left\{\gamma_{n}\right\} \subset[a, b] \subset\left(0, \frac{1}{\mu L}\right)$, for $L=\max \left\{L_{1}, L_{2}\right\}$ and $\left\{a_{n}\right\},\left\{b_{n}\right\},\left\{c_{n}\right\},\left\{d_{n}\right\} \subset[e, 1) \subset(0,1)$ such that $a_{n}+b_{n}+c_{n}+d_{n}=1$, converges strongly to $x^{*} \in V I\left(C, A_{1}\right) \cap V I\left(C, A_{2}\right) \cap F(T)$.

This now leads to the following important question.

Question. Can we obtain a strong convergence result for approximating a common element of the set of fixed points of a mapping, which is more general that the Bregman relatively nonexpansive mapping and the set of solutions of variational inequality problem with Lipschitz monotone mappings in spaces, which is beyond 2-uniformly convex and uniformly smooth real Banach spaces?

Motivated and inspired by the above results, it is our purpose in this paper to introduce the concept of a Bregman relatively $f$-nonexpansive mapping and construct an algorithm for approximating a common element of the set of $f$-fixed points of Bregman relatively $f$-nonexpansive mappings and the set of solutions of variational inequality problems with Lipschitz monotone mappings in the setting of real reflexive Banach spaces. Our results provide an affirmative answer to the question above. Our results improve, extend, and generalize many corresponding results in the literature. 


\section{Preliminaries}

In this section, we recall some known and useful results which will be used in the sequel.

Let $E$ be a real Banach space. $E$ is said to be strictly convex if $\left\|\frac{x+y}{2}\right\|<1$, whenever $x, y \in$ $S_{E}=\{x \in E:\|x\|=1\}$ and $x \neq y$. The norm of $E$ is said to be Gâteaux differentiable or smooth if

$$
\lim _{t \rightarrow 0} \frac{\|x+t y\|-\|x\|}{t}
$$

exists for each $x, y \in S_{E}$. For the rest of this section, let $E$ be a real reflexive Banach space with its dual $E^{*}$, and let $f: E \rightarrow(-\infty,+\infty]$ be a proper, convex and lower-semicontinuous function. We denote the domain of $f$ by $\operatorname{dom} f=\{x \in E: f(x)<\infty\}$. For any $x \in$ int dom $f$ and any $y \in E$, we denote by $f^{\prime}(x, y)$ the right-hand derivative of $f$ at $x$ in the direction of $y$, that is,

$$
f^{\prime}(x, y)=\lim _{t \rightarrow 0+} \frac{f(x+t y)-f(x)}{t} .
$$

The function $f$ is said to be Gâteaux differentiable at $x$ if

$$
\lim _{t \rightarrow 0} \frac{f(x+t y)-f(x)}{t},
$$

exists for any $y \in E$. In this case, the gradient of $f$ at $x$ is the linear function $\nabla f(x)$, which is defined by $\langle y, \nabla f(x)\rangle:=f^{\prime}(x, y)$ for all $y \in E$. The function $f$ is said to be Gâteaux differentiable if it is a Gâteaux differentiable at every point $x \in \operatorname{int} \operatorname{domf}$. When the subdifferential of $f$ is single-valued, it coincides with the gradient of $f, \partial f$, that is, $\partial f=\nabla f$ (see, e.g., [14]). Furthermore, $f$ is said to be uniformly Fréchet differentiable on a subset $C$ of $E$, if the limit in (2.1) is attained uniformly for $x \in C$ and $\|y\|=1$ and $f$ is called strongly coercive if $\lim _{\|x\| \rightarrow \infty} \frac{f(x)}{\|x\|}=\infty$.

The Fenchel conjugate of $f$ is a function $f^{*}: E^{*} \rightarrow(-\infty,+\infty]$, defined by $f^{*}\left(x^{*}\right)=\sup \left\{\left\langle x^{*}, x\right\rangle\right.$ $-f(x): x \in E\}$ for $x^{*} \in E^{*}$. The function $f$ is said to be Legendre if it satisfies the following conditions:

(i) the interior of the domain of $f$, int $\operatorname{dom} f$, is nonempty, $f$ is Gâteaux differentiable and $\operatorname{dom} \nabla f=\operatorname{int} \operatorname{dom} f$

(ii) the interior of the domain of $f^{*}$, int $\operatorname{dom} f^{*}$, is nonempty, $f^{*}$ is Gâteaux differentiable and $\operatorname{dom} \nabla f^{*}=$ int dom $f^{*}$.

One of the important and interesting Legendre function is $f(x)=\frac{1}{p}\|x\|^{p}(1<p<\infty)$ with the conjugate functions $f^{*}\left(x^{*}\right)=\frac{1}{q}\left\|x^{*}\right\|^{q}(1<q<\infty)$, (see, e.g., $[15,16]$ ), where $\frac{1}{p}+\frac{1}{q}=1$. In this case, the gradient $\nabla f$ coincides with the generalized duality mapping, $J_{p}$, of $E$, that is, $\nabla f=J_{p}$.

Definition 2.1. Let $f: E \rightarrow \mathbb{R}$, be a Gâteaux differentiable convex function. The function $D_{f}: \operatorname{dom} f \times$ int domf $\rightarrow[0, \infty)$ defined by

$$
D_{f}(y, x)=f(y)-f(x)-\langle\nabla f(x), y-x\rangle, x, y \in E .
$$

is called the Bregman distance with respect to $f$ [17].

We note that the Bregman distance has the following two important properties (see, e.g., $[18,19])$, the three point identity, for any $x \in \operatorname{dom} f$ and $y, z \in \operatorname{int} \operatorname{dom} f$,

$$
D_{f}(x, y)+D_{f}(y, z)-D_{f}(x, z)=\langle\nabla f(z)-\nabla f(y), x-y\rangle
$$


and the four point identity

$$
D_{f}(y, x)-D_{f}(y, z)-D_{f}(w, x)+D_{f}(w, z)=\langle\nabla f(z)-\nabla f(x), y-w\rangle .
$$

Lemma 2.1. [20, 21] If $f: E \rightarrow \mathbb{R}$ is a strongly coercive Legendre function, then

(i) $\nabla f: E \rightarrow E^{*}$ is one-to-one, onto and norm-to-weak ${ }^{*}$ continuous;

(ii) $\left\{x \in E: D_{f}(x, y) \leq r\right\}$ is bounded for all $y \in E$ and $r>0$;

(iii) dom $f^{*}=E^{*}, f^{*}$ is Gâteaux differentiable and $\nabla f^{*}=(\nabla f)^{-1}$.

Lemma 2.2. [14] If $f: E \rightarrow(-\infty,+\infty]$ is a proper, lower semi-continuous and convex function, then $f^{*}: E^{*} \rightarrow(-\infty,+\infty]$ is a proper, weak ${ }^{*}$ lower semi-continuous and convex function. Thus, for all $z \in E$, we have

$$
D_{f}\left(z, \nabla f^{*}\left(\sum_{i=1}^{N} t_{i} \nabla f\left(x_{i}\right)\right)\right) \leq \sum_{i=1}^{N} t_{i} D_{f}\left(z, x_{i}\right),
$$

where $\left\{x_{i}\right\} \subseteq E$ and $\left\{t_{i}\right\} \subseteq(0,1)$ with $\sum_{i=1}^{N} t_{i}=1$.

Lemma 2.3. [22] If $f: E \rightarrow \mathbb{R}$ is a uniformly Fréchet differentiable and bounded on bounded subsets of $E$, then $\nabla f$ is norm-to-norm uniformly continuous on bounded subsets of $E$ and hence both $f$ and $\nabla f$ are bounded on bounded subset of $E$.

Definition 2.2. Let $f: E \rightarrow(-\infty,+\infty]$ be a Gâteaux differentiable function. The function $v_{f}(x, t): E \times \mathbb{R}^{+} \rightarrow \mathbb{R}$ defined by

$$
v_{f}(x, t)=\inf _{\{y \in E:\|x-y\|=t\}} D_{f}(y, x),
$$

is called the Modulus of total convexity of $f$ at $x \in \operatorname{int} \operatorname{dom} f$. $f$ is said to be totally convex if $v_{f}(x, t)>0$, for all $x \in E$ and $t>0$.

We remark that $f$ is totally convex on bounded subsets if and only if $f$ is uniformly convex on bounded subsets of $E$ (see, e.g., [23, Theorem 2.10]). Note that if $f: E \rightarrow(-\infty, \infty]$ is a Legendre function and $E$ is a reflexive Banach space, then $\nabla f^{*}=(\nabla f)^{-1}$ (see, e.g., [24]). The Bregman projection with respect to $f$ on $x \in$ int dom $f$ onto a nonempty, closed and convex set $C \subseteq$ int domf is denoted by $P_{C}^{f}(x) \in C$, which satisfies

$$
D_{f}\left(P_{C}^{f}(x), x\right)=\inf \left\{D_{f}(y, x): y \in C\right\} .
$$

Remark 2.1. Some of the special cases of the Bregman projection are the following:

(i) If, in (2.4), $C$ is a closed and convex subset of a real reflexive Banach space $E$ and $f(x)=\|x\|^{2}$, then the Bregman projection $P_{C}^{f}(x)$ reduces to the generalized projection $\Pi_{C}(x)$, which is defined by

$$
\phi\left(\Pi_{C}(x), x\right)=\min _{y \in C} \phi(y, x),
$$

where $\phi(y, x)=\|y\|^{2}-2\langle y, J x\rangle+\|x\|^{2}$, and $J$ is the normalized duality mapping from $E$ into $2^{E^{*}}$.

(ii) If, in (2.4), $E=H$, a real Hilbert space and $f(x)=\|x\|^{2}$, then the Bregman projection $P_{C}^{f}(x)$ reduces to the metric projection of $x$ onto $C$. 
Lemma 2.4. [23] Let $f: E \rightarrow \mathbb{R}$ be a Gâteaux differentiable and totally convex function and let $x \in E$. The Bregman projection $P_{C}^{f}$ from $E$ onto $C$, where $C$ is a nonempty, closed and convex subset of $E$, is a unique vector with the following properties:

(i) $z=P_{C}^{f}(x)$ if and only if $\langle\nabla f(x)-\nabla f(z), y-z\rangle \leq 0, \forall y \in C$.

(ii) $D_{f}\left(y, P_{C}^{f}(x)\right)+D_{f}\left(P_{C}^{f}(x), x\right) \leq D_{f}(y, x)$, for all $y \in C$ and $x \in E$.

Let $f: E \rightarrow \mathbb{R}$ be a Legendre function. Let $V_{f}: E \times E^{*} \rightarrow[0,+\infty)$, associated with $f$, be defined by

$$
V_{f}\left(x, x^{*}\right)=f(x)-\left\langle x, x^{*}\right\rangle+f^{*}\left(x^{*}\right), \quad \forall x \in E, x^{*} \in E^{*} .
$$

Then, $V_{f}$ is nonnegative with the following properties (see, e.g., [25])

$$
V_{f}\left(x, x^{*}\right)=D_{f}\left(x, \nabla f^{*}\left(x^{*}\right)\right), \quad \forall x \in E, x^{*} \in E^{*},
$$

and

$$
V_{f}\left(x, x^{*}\right)+\left\langle y^{*}, \nabla f^{*}\left(x^{*}\right)-x\right\rangle \leq V_{f}\left(x, x^{*}+y^{*}\right), \quad \forall x \in E, x^{*}, y^{*} \in E^{*} .
$$

Let $C$ be nonempty, closed and convex subset of $E$. Let $T: C \rightarrow E$ be a mapping. A point $x \in C$ is called an asymptotic fixed point of $T$ if $C$ contains a sequence $\left\{x_{n}\right\}$ which converges weakly to $x$ and $\lim _{n \rightarrow \infty}\left\|T x_{n}-x_{n}\right\|=0$. We denote the set of asymptotic fixed points of $T$ by $\widehat{F}(T)$.

Definition 2.3. [26] Let $C$ be a nonempty, closed and convex subset of $E$. A mapping $T: C \rightarrow E$ is called relatively nonexpansive if

(i) $F(T) \neq \emptyset$;

(ii) $\phi(p, T y) \leq \phi(p, y) \forall y \in C, p \in F(T)$;

(iii) $\widehat{F}(T)=F(T)$.

Definition 2.4. [27] Let $C$ be a nonempty, closed and convex subset of $E$ and let $T: C \rightarrow E^{*}$ be a map. A point $p \in C$ is called an asymptotic $J$-fixed point of $T$ if $C$ contains a sequence $\left\{x_{n}\right\}$ which converges weakly to $p$ and $\lim _{n \rightarrow \infty}|| T x_{n}-J x_{n} \|=0$.

We denote the set of asymptotic $J$-fixed points of $T$ by $\widehat{F}_{J}(T)$.

Definition 2.5. [27] Let $C$ be a nonempty, closed and convex subset of $E$. A mapping $T: C \rightarrow E^{*}$ is called relatively $J$-nonexpansive if

(i) $\widehat{F}_{J}(T)=F(T) \neq \emptyset$;

(ii) $\phi\left(p, J^{-1} T y\right) \leq \phi(p, y) \forall y \in C, p \in \widehat{F}_{J}(T)$.

Definition 2.6. [28] A Gâteaux differentiable function $f$ is called strongly convex with constant $\mu>0$ if the following inequality holds:

$$
\langle\nabla f(x)-\nabla f(y), x-y\rangle \geq \mu\|x-y\|^{2},
$$

for all points $x, y$ in its domain, or equivalently (see, e.g., [29])

$$
f(y) \geq f(x)+\langle\nabla f(x), y-x\rangle+\frac{\mu}{2}\|x-y\|^{2} .
$$

If $E$ is a smooth and strictly convex Banach space, then $f(x)=\|x\|^{2}$ is an example of a strongly convex function with parameter $0<\mu \leq 2$. 
Definition 2.7. [21] A function $f$ is called uniformly convex function with modulus $\phi$ if, for each $x, y$ in its domain and $t \in[0,1]$,

$$
f(t x+(1-t) y) \leq t f(x)+(1-t) f(y)-t(1-t) \phi(\|x-y\|),
$$

where $\phi$ is a function that is increasing and vanishes only at 0 .

Lemma 2.5. Let $f$ be a convex and lower semi-continuous function on a Banach space E. The following assertions are equivalent (see, e.g., [21]):

(i) $f$ is uniformly convex;

(ii) There exists modulus $\phi, \forall\left(x, x^{*}\right),\left(y, y^{*}\right) \in G p h(\partial f)$ such that

$$
f(y) \geq f(x)+\left\langle y-x, x^{*}\right\rangle+\phi(\|x-y\|) ;
$$

(iii) dom $f^{*}=E^{*}, f^{*}$ is Frèchet differentiable and $\nabla f^{*}$ is uniformly continuous.

Note that a strongly convex function is uniformly convex with $\phi(\alpha)=\frac{\mu}{2} \alpha^{2}$ and hence the class of uniformly convex functions contains the class of strongly convex functions.

Lemma 2.6. [30] Let $E$ be a Banach space and let $r>0$ be a constant. Let $f: E \rightarrow \mathbb{R}$ be a continuous and convex function, which is uniformly convex on bounded subset of $E$. Then

$$
f\left(\sum_{k=0}^{n} \alpha_{k} x_{k}\right) \leq \sum_{k=0}^{n} \alpha_{k} f\left(x_{k}\right)-\alpha_{i} \alpha_{j} \rho_{r}\left(|| x_{i}-x_{j}||\right),
$$

for all $0 \leq i, j \leq n, x_{k} \in B_{r}, \alpha_{k} \in(0,1)$ with $\sum_{k=0}^{n} \alpha_{k}=1$, where $\rho_{r}$ is the gauge of uniform convexity of $f$.

Lemma 2.7. [21] Let $f: E \rightarrow \mathbb{R}$ be a continuous convex function on a reflexive Banach space $E$, which is strongly coercive. Then the following statements are equivalent:

(i) $f$ is bounded on bounded subset and uniformly smooth on bounded subset of $E$;

(ii) $f^{*}$ is Fréchet differentiable and $f^{*}$ is uniformly norm-to-norm continuous on bounded subset of $E^{*}$;

(iii) dom $f^{*}=E^{*}, f^{*}$ is strongly coercive and uniformly convex on bounded subset of $E^{*}$.

Lemma 2.8. [30] Let $f: E \rightarrow \mathbb{R}$ be a Gâteaux differentiable function, which is uniformly convex on bounded subset of $E$. Let $\left\{x_{n}\right\}$ and $\left\{y_{n}\right\}$ be bounded sequence in E. Then the following statements are equivalent:

(i) $\lim _{n \rightarrow \infty} D_{f}\left(x_{n}, y_{n}\right)=0$;

(ii) $\lim _{n \rightarrow \infty}|| x_{n}-y_{n} \|=0$.

Lemma 2.9. [31] Let $f$ be a strongly convex function with constant $\mu>0$. Then, for all $y \in$ dom $f$ and $x \in$ int dom $f$,

$$
D_{f}(y, x) \geq \frac{\mu}{2}\|x-y\|^{2}
$$

where $D_{f}(y, x)$ is a Bregman distance with respect to $f$.

Lemma 2.10. [32] Let $\left\{a_{n}\right\}$ be a sequence of nonnegative real numbers satisfying the following relation: $a_{n+1} \leq\left(1-\alpha_{n}\right) a_{n}+\alpha_{n} \delta_{n}, n \geq n_{0}$, where $\left\{\alpha_{n}\right\} \subset(0,1)$ and $\left\{\delta_{n}\right\} \subset \mathbb{R}$ satisfying the following conditions: $\sum_{n=1}^{\infty} \alpha_{n}=\infty$, and $\limsup _{n \rightarrow \infty} \delta_{n} \leq 0$. Then, $\lim _{n \rightarrow \infty} a_{n}=0$. 
Lemma 2.11. [33] Let $\left\{s_{k}\right\}$ be a sequence of real numbers that does not decrease at infinity, in the sense that there exists a subsequence $\left\{s_{k_{j}}\right\}$ of $\left\{s_{k}\right\}$ such that $s_{k_{j}}<s_{k_{j}+1}$ for all $j \geq 0$. Define an integer sequence $\left\{m_{k}\right\}_{k \geq k_{0}}$ as $m_{k}=\max \left\{k_{0} \leq l \leq k: s_{l}<s_{l+1}\right\}$. Then $m_{k} \rightarrow \infty$ as $k \rightarrow \infty$ and for all $k \geq k_{0} \max \left\{s_{m_{k}}, s_{k}\right\} \leq s_{m_{k}+1}$.

\section{MAIN RESULTS}

From now on, $E$ is assumed to be a real reflexive Banach space with its dual $E^{*}, C$ is a nonempty, closed and convex subset of $E$, and $f: E \rightarrow(-\infty,+\infty]$ is a proper, continuous and convex function on a real Banach space $E$. We denote the Fenchel conjugate of $f$ by $f^{*}: E^{*} \rightarrow$ $(-\infty,+\infty]$ and the family of such functions by $\mathscr{F}(E)$. Let $\left\{\alpha_{n}\right\} \subset(0,1)$ be a real sequence satisfying $\lim _{n \rightarrow \infty} \alpha_{n}=0$, and $\sum_{n=1}^{\infty} \alpha_{n}=\infty$.

Definition 3.1. Let $C$ be a nonempty, closed and convex subset of $E$. Let $T: C \rightarrow E^{*}$ be a mapping. A point $p \in C$ is said to be a $f$-fixed point of $T$ if $T p=\nabla f p$.

We denote the set of $f$-fixed points of $T$ by $F_{f}(T)$, that is, $F_{f}(T):=\{p \in C: T p=\nabla f p\}$.

Definition 3.2. Let $T: C \rightarrow E^{*}$ be a mapping. A point $p \in C$ is said to be an $f$-asymptotic fixed point of $T$ if $C$ contains a sequence $\left\{x_{n}\right\}$ which converges weakly to $p$ and $\lim _{n \rightarrow \infty} \| \nabla f x_{n}-$ $T x_{n} \|=0$.

We denote the set of $f$-asymptotic fixed points of $T$ by $\widehat{F}_{f}(T)$.

Definition 3.3. A mapping $T: C \rightarrow E^{*}$ is said to be Bregman relatively $f$-nonexpansive if

(i) $F_{f}(T) \neq \emptyset$;

(ii) $D_{f}\left(p, \nabla f^{*} T y\right) \leq D_{f}(p, y), \forall y \in C, p \in \widehat{F}_{f}(T)$;

(iii) $\widehat{F}_{f}(T)=F_{f}(T)$.

First, we prove the following lemmas.

Lemma 3.1. If $T: C \rightarrow E^{*}$ is a Bregman relatively $f$-nonexpansive mapping, then $F_{f}(T)$ is closed and convex.

Proof. We first prove that $F_{f}(T)$ is closed. Let $\left\{x_{n}\right\}$ be a sequence in $F_{f}(T)$ such that $x_{n} \rightarrow p \in$ $C$. From the definition of $T$, we have $D_{f}\left(x_{n}, \nabla f^{*} T p\right) \leq D_{f}\left(x_{n}, p\right)$, for each $n \geq 0$. Thus,

$$
D_{f}\left(p, \nabla f^{*} T p\right)=\lim _{n \rightarrow \infty} D_{f}\left(x_{n}, \nabla f^{*} T p\right) \leq \lim _{n \rightarrow \infty} D_{f}\left(x_{n}, p\right)=D_{f}(p, p)=0,
$$

which implies that $p=\nabla f^{*} T p$. Hence, $\nabla f p=T p$. So, we have $p \in F_{f}(T)$, that is, $F_{f}(T)$ is closed.

Next, we show that $F_{f}(T)$ is convex. Let $p, q \in F_{f}(T)$ and $w=t p+(1-t) q$, for $t \in(0,1)$. Then, from (2.2) and the definition of $T$, we have

$$
\begin{aligned}
D_{f}\left(w, \nabla f^{*} T w\right)= & f(w)-\left\langle t p+(1-t) q-\nabla f^{*} T w, T w\right\rangle-f\left(\nabla f^{*} T w\right) \\
= & f(w)-t\left\langle p-\nabla f^{*} T w, T w\right\rangle-(1-t)\left\langle q-\nabla f^{*} T w, T w\right\rangle-f\left(\nabla f^{*} T w\right) \\
= & f(w)+t f(p)-t f(p)-t\left\langle p-\nabla f^{*} T w, T w\right\rangle+(1-t) f(q)-(1-t) f(q) \\
& -(1-t)\left\langle q-\nabla f^{*} T w, T w\right\rangle-f\left(\nabla f^{*} T w\right) \\
= & f(w)+t D_{f}\left(p, \nabla f^{*} T w\right)+(1-t) D_{f}\left(q, \nabla f^{*} T w\right)-t f(p)-(1-t) f(q) \\
\leq & f(w)+t D_{f}(p, w)+(1-t) D_{f}(q, w)-t f(p)-(1-t) f(q) \\
= & f(w)-\langle w-w, \nabla f w\rangle-f(w)=0 .
\end{aligned}
$$


This impels $w=\nabla f^{*} T w$. Hence $\nabla f w=T w$ and $w \in F_{f}(T)$, that is, $F_{f}(T)$ is convex.

Theorem 3.1. Let $f \in \mathscr{F}(E)$ be a strongly coercive, bounded and uniformly Fréchet differentiable Legendre function, which is strongly convex with constant $\mu>0$ on bounded subsets of $E$. Let $A_{i}: C \rightarrow E^{*}$ be a $L_{i}$-Lipschitz monotone mapping with constant $L_{i}$, for $i=1,2$ and let $T: C \rightarrow E^{*}$ be a Bregman relatively $f$-nonexpansive mapping such that $\Omega=V I\left(C, A_{1}\right) \cap$ $\operatorname{VI}\left(C, A_{2}\right) \cap F_{f}(T) \neq \emptyset$. For arbitrary $x_{0}, w \in C$, define an iterative sequence by

$$
\left\{\begin{array}{l}
y_{n}=P_{C}^{f} \nabla f^{*}\left(\nabla f x_{n}-\gamma_{n} A_{1} x_{n}\right), \\
z_{n}=P_{C}^{f} \nabla f^{*}\left(\nabla f x_{n}-\gamma_{n} A_{2} x_{n}\right), \\
w_{n}=\nabla f^{*}\left(\beta_{n} \nabla f x_{n}+\delta_{n} T x_{n}+\eta_{n} \nabla f u_{n}+\theta_{n} \nabla f v_{n}\right), \\
x_{n+1}=P_{C}^{f} \nabla f^{*}\left(\alpha_{n} \nabla f w+\left(1-\alpha_{n}\right) \nabla f w_{n}\right),
\end{array}\right.
$$

where $u_{n}=P_{C}^{f} \nabla f^{*}\left(\nabla f x_{n}-\gamma_{n} A_{1} y_{n}\right), v_{n}=P_{C}^{f} \nabla f^{*}\left(\nabla f x_{n}-\gamma_{n} A_{2} z_{n}\right), 0<\underline{\gamma} \leq \gamma_{n} \leq \bar{\gamma}<\frac{\mu}{L}$, for $L:=\max \left\{L_{1}, L_{2}\right\}$ and $\left\{\beta_{n}\right\},\left\{\delta_{n}\right\},\left\{\eta_{n}\right\},\left\{\theta_{n}\right\} \subset[\varepsilon, 1) \subset(0,1)$ such that $\beta_{n}+\delta_{n}+\eta_{n}+\theta_{n}=1$, for all $n \geq 0$. Then, $\left\{x_{n}\right\}$ is bounded.

Proof. Let $q \in \Omega$. Now, from (3.1), Lemma 2.4 (ii), (2.2), (1.1) and the monotonicity of $A_{1}$, we have

$$
\begin{aligned}
D_{f}\left(q, u_{n}\right) \leq & D_{f}\left(q, \nabla f^{*}\left(\nabla f x_{n}-\gamma_{n} A_{1} y_{n}\right)\right)-D_{f}\left(u_{n}, \nabla f^{*}\left(\nabla f x_{n}-\gamma_{n} A_{1} y_{n}\right)\right) \\
= & f(q)-\left\langle q-\nabla f^{*}\left(\nabla f x_{n}-\gamma_{n} A_{1} y_{n}\right), \nabla f x_{n}-\gamma_{n} A_{1} y_{n}\right\rangle \\
& -f\left(\nabla f^{*}\left(\nabla f x_{n}-\gamma_{n} A_{1} y_{n}\right)\right)-f\left(u_{n}\right) \\
& +\left\langle u_{n}-\nabla f^{*}\left(\nabla f x_{n}-\gamma_{n} A_{1} y_{n}\right), \nabla f x_{n}-\gamma_{n} A_{1} y_{n}\right\rangle \\
& +f\left(\nabla f^{*}\left(\nabla f x_{n}-\gamma_{n} A_{1} y_{n}\right)\right) \\
= & f(q)+\left\langle u_{n}-q, \nabla f x_{n}\right\rangle-\left\langle u_{n}-q, \gamma_{n} A_{1} y_{n}\right\rangle-f\left(u_{n}\right) \\
= & f(q)-\left\langle q-x_{n}, \nabla f x_{n}\right\rangle-f\left(x_{n}\right)+\left\langle q-x_{n}, \nabla f x_{n}\right\rangle+f\left(x_{n}\right) \\
& +\left\langle u_{n}-q, \nabla f x_{n}\right\rangle-\left\langle u_{n}-q, \gamma_{n} A_{1} y_{n}\right\rangle-f\left(u_{n}\right) \\
= & D_{f}\left(q, x_{n}\right)+\left\langle u_{n}-x_{n}, \nabla f x_{n}\right\rangle+f\left(x_{n}\right)-f\left(u_{n}\right)-\left\langle u_{n}-q, \gamma_{n} A_{1} y_{n}\right\rangle
\end{aligned}
$$

and

$$
\begin{aligned}
D_{f}\left(q, u_{n}\right) \leq & D_{f}\left(q, x_{n}\right)-D_{f}\left(u_{n}, x_{n}\right)-\left\langle u_{n}-q, \gamma_{n} A_{1} y_{n}\right\rangle \\
= & D_{f}\left(q, x_{n}\right)-D_{f}\left(u_{n}, x_{n}\right)+\gamma_{n}\left\langle q-y_{n}, A_{1} y_{n}-A_{1} q\right\rangle \\
& +\gamma_{n}\left\langle q-y_{n}, A_{1} q\right\rangle+\left\langle y_{n}-u_{n}, \gamma_{n} A_{1} y_{n}\right\rangle \\
\leq & D_{f}\left(q, x_{n}\right)-D_{f}\left(u_{n}, x_{n}\right)+\left\langle y_{n}-u_{n}, \gamma_{n} A_{1} y_{n}\right\rangle .
\end{aligned}
$$

In addition, we from (2.3) that

$$
D_{f}\left(u_{n}, x_{n}\right)=D_{f}\left(u_{n}, y_{n}\right)+D_{f}\left(y_{n}, x_{n}\right)+\left\langle\nabla f x_{n}-\nabla f y_{n}, y_{n}-u_{n}\right\rangle .
$$

Substituting (3.3) into (3.2), we obtain

$$
\begin{aligned}
D_{f}\left(q, u_{n}\right) \leq & D_{f}\left(q, x_{n}\right)-D_{f}\left(u_{n}, y_{n}\right)-D_{f}\left(y_{n}, x_{n}\right) \\
& -\left\langle\nabla f x_{n}-\nabla f y_{n}, y_{n}-u_{n}\right\rangle+\left\langle y_{n}-u_{n}, \gamma_{n} A_{1} y_{n}\right\rangle \\
= & D_{f}\left(q, x_{n}\right)-D_{f}\left(u_{n}, y_{n}\right)-D_{f}\left(y_{n}, x_{n}\right) \\
& +\left\langle y_{n}-u_{n}, \gamma_{n} A_{1} y_{n}+\nabla f y_{n}-\nabla f x_{n}\right\rangle .
\end{aligned}
$$


From Lemma 2.4 (i), we have

$$
\begin{aligned}
\left\langle y_{n}-u_{n}, \gamma_{n} A_{1} y_{n}+\nabla f y_{n}-\nabla f x_{n}\right\rangle= & \left\langle u_{n}-y_{n}, \gamma_{n} A_{1} x_{n}-\gamma_{n} A_{1} y_{n}\right\rangle \\
& +\left\langle u_{n}-y_{n}, \nabla f x_{n}-\gamma_{n} A_{1} x_{n}-\nabla f y_{n}\right\rangle \\
\leq & \left\langle u_{n}-y_{n}, \gamma_{n} A_{1} x_{n}-\gamma_{n} A_{1} y_{n}\right\rangle .
\end{aligned}
$$

Furthermore, (3.4), (3.5), Lemma 2.9 and the fact that $A_{1}$ is $L_{1}$-Lipschitz, we obtain

$$
\begin{aligned}
D_{f}\left(q, u_{n}\right) \leq & D_{f}\left(q, x_{n}\right)-D_{f}\left(u_{n}, y_{n}\right)-D_{f}\left(y_{n}, x_{n}\right)+\left\langle u_{n}-y_{n}, \gamma_{n} A_{1} x_{n}-\gamma_{n} A_{1} y_{n}\right\rangle \\
\leq & D_{f}\left(q, x_{n}\right)-D_{f}\left(u_{n}, y_{n}\right)-D_{f}\left(y_{n}, x_{n}\right)+\gamma_{n}\left\|u_{n}-y_{n}\right\|\left\|A_{1} x_{n}-A_{1} y_{n}\right\| \\
\leq & D_{f}\left(q, x_{n}\right)-D_{f}\left(u_{n}, y_{n}\right)-D_{f}\left(y_{n}, x_{n}\right)+\gamma_{n} L\left\|u_{n}-y_{n}\right\|\left\|x_{n}-y_{n}\right\| \\
\leq & D_{f}\left(q, x_{n}\right)-D_{f}\left(u_{n}, y_{n}\right)-D_{f}\left(y_{n}, x_{n}\right)+\frac{\gamma_{n} L}{2}\left[\left\|u_{n}-y_{n}\right\|^{2}+\left\|x_{n}-y_{n}\right\|^{2}\right] \\
\leq & D_{f}\left(q, x_{n}\right)-\frac{\mu}{2}\left\|u_{n}-y_{n}\right\|^{2}-\frac{\mu}{2}\left\|y_{n}-x_{n}\right\|^{2} \\
& +\frac{\gamma_{n} L}{2}\left[\left\|u_{n}-y_{n}\right\|^{2}+\left\|x_{n}-y_{n}\right\|^{2}\right] \\
= & D_{f}\left(q, x_{n}\right)-\left(\frac{\mu-\gamma_{n} L}{2}\right)\left[\left\|u_{n}-y_{n}\right\|^{2}+\left\|x_{n}-y_{n}\right\|^{2}\right] .
\end{aligned}
$$

Similarly, we obtain that

$$
D_{f}\left(q, v_{n}\right) \leq D_{f}\left(q, x_{n}\right)-\left(\frac{\mu-\gamma_{n} L}{2}\right)\left[\left\|v_{n}-z_{n}\right\|^{2}+\left\|x_{n}-z_{n}\right\|^{2}\right]
$$

Again, from (2.5), (3.1), (2.6) and Lemma 2.6, we get

$$
\begin{aligned}
D_{f}\left(q, w_{n}\right)= & V_{f}\left(q, \beta_{n} \nabla f x_{n}+\delta_{n} T x_{n}+\eta_{n} \nabla f u_{n}+\theta_{n} \nabla f v_{n}\right) \\
= & f(q)-\left\langle q, \beta_{n} \nabla f x_{n}+\delta_{n} T x_{n}+\eta_{n} \nabla f u_{n}+\theta_{n} \nabla f v_{n}\right\rangle \\
& +f^{*}\left(\beta_{n} \nabla f x_{n}+\delta_{n} T x_{n}+\eta_{n} \nabla f u_{n}+\theta_{n} \nabla f v_{n}\right) \\
\leq & \beta_{n} f(q)+\delta_{n} f(q)+\eta_{n} f(q)+\theta_{n} f(q) \\
& -\left\langle q, \beta_{n} \nabla f x_{n}\right\rangle-\left\langle q, \delta_{n} T x_{n}\right\rangle-\left\langle q, \eta_{n} \nabla f u_{n}\right\rangle-\left\langle q, \theta_{n} \nabla f v_{n}\right\rangle \\
& +\beta_{n} f^{*}\left(\nabla f x_{n}\right)+\delta_{n} f^{*}\left(T x_{n}\right)+\eta_{n} f^{*}\left(\nabla f u_{n}\right)+\theta_{n} f^{*}\left(\nabla f v_{n}\right) \\
& -\beta_{n} \delta_{n} \rho_{r}^{*}\left(\left\|\nabla f x_{n}-T x_{n}\right\|\right) \\
= & \beta_{n} V_{f}\left(q, \nabla f x_{n}\right)+\delta_{n} V_{f}\left(q, T x_{n}\right)+\eta_{n} V_{f}\left(q, \nabla f u_{n}\right) \\
& +\theta_{n} V_{f}\left(q, \nabla f v_{n}\right)-\beta_{n} \delta_{n} \rho_{r}^{*}\left(\left\|\nabla f x_{n}-T x_{n}\right\|\right) \\
= & \beta_{n} D_{f}\left(q, x_{n}\right)+\delta_{n} D_{f}\left(q, \nabla f^{*} T x_{n}\right)+\eta_{n} D_{f}\left(q, u_{n}\right)+\theta_{n} D_{f}\left(q, v_{n}\right) \\
& -\beta_{n} \delta_{n} \rho_{r}^{*}\left(\left\|\nabla f x_{n}-T x_{n}\right\|\right) .
\end{aligned}
$$


Using the assumption on $T$, inequalities (3.6) and (3.7), we obtain

$$
\begin{aligned}
D_{f}\left(q, w_{n}\right) \leq & \beta_{n} D_{f}\left(q, x_{n}\right)+\delta_{n} D_{f}\left(q, x_{n}\right)+\eta_{n} D_{f}\left(q, u_{n}\right)+\theta_{n} D_{f}\left(q, v_{n}\right) \\
& -\beta_{n} \delta_{n} \rho_{r}^{*}\left(\left\|\nabla f x_{n}-T x_{n}\right\|\right) \\
\leq & \beta_{n} D_{f}\left(q, x_{n}\right)+\delta_{n} D_{f}\left(q, x_{n}\right) \\
& +\eta_{n}\left[D_{f}\left(q, x_{n}\right)-\left(\frac{\mu-\gamma_{n} L}{2}\right)\left(\left\|u_{n}-y_{n}\right\|^{2}+\left\|x_{n}-y_{n}\right\|^{2}\right)\right] \\
& +\theta_{n}\left[D_{f}\left(q, x_{n}\right)-\left(\frac{\mu-\gamma_{n} L}{2}\right)\left(\left\|v_{n}-z_{n}\right\|^{2}+\left\|x_{n}-z_{n}\right\|^{2}\right)\right] \\
& -\beta_{n} \delta_{n} \rho_{r}^{*}\left(\left\|\nabla f x_{n}-T x_{n}\right\|\right) \\
= & D_{f}\left(q, x_{n}\right)-\eta_{n}\left[\left(\frac{\mu-\gamma_{n} L}{2}\right)\left(\left\|u_{n}-y_{n}\right\|^{2}+\left\|x_{n}-y_{n}\right\|^{2}\right)\right] \\
& -\theta_{n}\left[\left(\frac{\mu-\gamma_{n} L}{2}\right)\left(\left\|v_{n}-z_{n}\right\|^{2}+\left\|x_{n}-z_{n}\right\|^{2}\right)\right] \\
& -\beta_{n} \delta_{n} \rho_{r}^{*}\left(\left\|\nabla f x_{n}-T x_{n}\right\|\right) \\
\leq & D_{f}\left(q, x_{n}\right) .
\end{aligned}
$$

Now, from (3.1), (3.8), Lemma 2.4 (ii) and Lemma 2.2, we obtain

$$
\begin{aligned}
D_{f}\left(q, x_{n+1}\right) & =D_{f}\left(q, \nabla f^{*}\left(\alpha_{n} \nabla f w+\left(1-\alpha_{n}\right) \nabla f w_{n}\right)\right) \\
& \leq \alpha_{n} D_{f}(q, w)+\left(1-\alpha_{n}\right) D_{f}\left(q, w_{n}\right) \\
& \leq \alpha_{n} D_{f}(q, w)+\left(1-\alpha_{n}\right) D_{f}\left(q, x_{n}\right) \\
& \leq \max \left\{D_{f}(q, w), D_{f}\left(q, x_{n}\right)\right\} .
\end{aligned}
$$

It follows that $D_{f}\left(q, x_{n}\right) \leq \max \left\{D_{f}(q, w), D_{f}\left(q, x_{0}\right)\right\}$. Thus, $\left\{D_{f}\left(q, x_{n}\right)\right\}$ is bounded. Therefore, by Lemma 2.1, we have that $\left\{x_{n}\right\}$ is bounded, so are $\left\{y_{n}\right\},\left\{z_{n}\right\},\left\{u_{n}\right\},\left\{w_{n}\right\}$ and $\left\{v_{n}\right\}$.

Next, we prove the main theorem of this section.

Theorem 3.2. Let $f \in \mathscr{F}(E)$ be a strongly coercive, bounded and uniformly Fréchet differentiable Legendre function, which is strongly convex with constant $\mu>0$ on bounded subsets of $E$. Let $A_{i}: C \rightarrow E^{*}$ be a $L_{i}$-Lipschitz monotone mapping with constant $L_{i}$, for $i=1,2$ and let $T: C \rightarrow E^{*}$ be a Bregman relatively $f$-nonexpansive mapping such that $\Omega=V I\left(C, A_{1}\right) \cap$ $\operatorname{VI}\left(C, A_{2}\right) \cap F_{f}(T) \neq \emptyset$. For arbitrary $x_{0}, w \in C$, define an iterative sequence by

$$
\left\{\begin{array}{l}
y_{n}=P_{C}^{f} \nabla f^{*}\left(\nabla f x_{n}-\gamma_{n} A_{1} x_{n}\right), \\
z_{n}=P_{C}^{f} \nabla f^{*}\left(\nabla f x_{n}-\gamma_{n} A_{2} x_{n}\right), \\
w_{n}=\nabla f^{*}\left(\beta_{n} \nabla f x_{n}+\delta_{n} T x_{n}+\eta_{n} \nabla f u_{n}+\theta_{n} \nabla f v_{n}\right), \\
x_{n+1}=P_{C}^{f} \nabla f^{*}\left(\alpha_{n} \nabla f w+\left(1-\alpha_{n}\right) \nabla f w_{n}\right),
\end{array}\right.
$$

where $u_{n}=P_{C}^{f} \nabla f^{*}\left(\nabla f x_{n}-\gamma_{n} A_{1} y_{n}\right), v_{n}=P_{C}^{f} \nabla f^{*}\left(\nabla f x_{n}-\gamma_{n} A_{2} z_{n}\right), 0<\underline{\gamma} \leq \gamma_{n} \leq \bar{\gamma}<\frac{\mu}{L}$, for $L:=\max \left\{L_{1}, L_{2}\right\}$ and $\left\{\beta_{n}\right\},\left\{\delta_{n}\right\},\left\{\eta_{n}\right\},\left\{\theta_{n}\right\} \subset[\varepsilon, 1) \subset(0,1)$ such that $\beta_{n}+\delta_{n}+\eta_{n}+\theta_{n}=1$, for all $n \geq 0$. Then, $\left\{x_{n}\right\}$ converges strongly to an element $x^{*}=P_{\Omega}^{f}(w)$.

Proof. From Theorem 3.1, we know that $\left\{x_{n}\right\}$ is bounded. Let $q=P_{\Omega}^{f}(w)$ and $r_{n}=\nabla f^{*}\left(\alpha_{n} \nabla f w+\right.$ $\left.\left(1-\alpha_{n}\right) \nabla f w_{n}\right)$. In view of Lemma 2.4 (i), we get $\langle u-q, \nabla f w-\nabla f q\rangle \leq 0, \forall u \in \Omega$. Now, from 
(2.6), (3.9) and Lemma 2.4, we get

$$
\begin{aligned}
D_{f}\left(q, x_{n+1}\right) \leq & D_{f}\left(q, \nabla f^{*}\left(\alpha_{n} \nabla f w+\left(1-\alpha_{n}\right) \nabla f w_{n}\right)\right) \\
= & V_{f}\left(q, \alpha_{n} \nabla f w+\left(1-\alpha_{n}\right) \nabla f w_{n}\right) \\
\leq & V_{f}\left(q, \alpha_{n} \nabla f w+\left(1-\alpha_{n}\right) \nabla f w_{n}-\alpha_{n}(\nabla f w-\nabla f q)\right) \\
& +\left\langle\alpha_{n}(\nabla f w-\nabla f q), r_{n}-q\right\rangle \\
= & D_{f}\left(q, \nabla f^{*}\left(\alpha_{n} \nabla f q+\left(1-\alpha_{n}\right) \nabla f w_{n}\right)\right)+\alpha_{n}\left\langle\nabla f w-\nabla f q, r_{n}-q\right\rangle .
\end{aligned}
$$

From Lemma 2.2 and (3.8), we arrive at

$$
\begin{aligned}
D_{f}\left(q, x_{n+1}\right) \leq & \alpha_{n} D_{f}(q, q)+\left(1-\alpha_{n}\right) D_{f}\left(q, w_{n}\right) \\
& +\alpha_{n}\left\langle\nabla f w-\nabla f q, r_{n}-q\right\rangle \\
= & \left(1-\alpha_{n}\right) D_{f}\left(q, w_{n}\right)+\alpha_{n}\left\langle\nabla f w-\nabla f q, r_{n}-q\right\rangle \\
\leq & \left(1-\alpha_{n}\right) D_{f}\left(q, x_{n}\right)+\alpha_{n}\left\langle\nabla f w-\nabla f q, r_{n}-q\right\rangle \\
\leq & \left(1-\alpha_{n}\right) D_{f}\left(q, x_{n}\right)+\alpha_{n}\|\nabla f w-\nabla f q\|\left\|r_{n}-x_{n}\right\| \\
& +\alpha_{n}\left\langle\nabla f w-\nabla f q, x_{n}-q\right\rangle .
\end{aligned}
$$

On the other hand, (3.9), Lemma 2.4 and Lemma 2.2 yield that

$$
\begin{aligned}
D_{f}\left(q, x_{n+1}\right) & =D_{f}\left(q, P_{C}^{f} \nabla f^{*}\left(\alpha_{n} \nabla f w+\left(1-\alpha_{n}\right) \nabla f w_{n}\right)\right) \\
& =D_{f}\left(q, \nabla f^{*}\left(\alpha_{n} \nabla f w+\left(1-\alpha_{n}\right) \nabla f w_{n}\right)\right) \\
& \leq \alpha_{n} D_{f}(q, w)+\left(1-\alpha_{n}\right) D_{f}\left(q, w_{n}\right) .
\end{aligned}
$$

By using (3.8), we obtain

$$
\begin{aligned}
D_{f}\left(q, x_{n+1}\right) \leq & \alpha_{n} D_{f}(q, w)+\left(1-\alpha_{n}\right) D_{f}\left(q, x_{n}\right) \\
& -\left(1-\alpha_{n}\right) \eta_{n}\left[\left(\frac{\mu-\gamma_{n} L}{2}\right)\left(\left\|u_{n}-y_{n}\right\|^{2}+\left\|x_{n}-y_{n}\right\|^{2}\right)\right] \\
& -\left(1-\alpha_{n}\right) \theta_{n}\left[\left(\frac{\mu-\gamma_{n} L}{2}\right)\left(\left\|v_{n}-z_{n}\right\|^{2}+\left\|x_{n}-z_{n}\right\|^{2}\right)\right] \\
& -\beta_{n} \delta_{n} \rho_{r}^{*}\left(\left\|\nabla f x_{n}-T x_{n}\right\|\right),
\end{aligned}
$$

which implies that

$$
\begin{aligned}
(1 & \left.-\alpha_{n}\right) \eta_{n}\left[\left(\frac{\mu-\gamma_{n} L}{2}\right)\left(\left\|u_{n}-y_{n}\right\|^{2}+\left\|x_{n}-y_{n}\right\|^{2}\right)\right] \\
& +\left(1-\alpha_{n}\right) \theta_{n}\left[\left(\frac{\mu-\gamma_{n} L}{2}\right)\left(\left\|v_{n}-z_{n}\right\|^{2}+\left\|x_{n}-z_{n}\right\|^{2}\right)\right] \\
& +\beta_{n} \delta_{n} \rho_{r}^{*}\left(\left\|\nabla f x_{n}-T x_{n}\right\|\right) \\
\leq & \alpha_{n}\left(D_{f}(q, w)-D_{f}\left(q, x_{n}\right)\right)+D_{f}\left(q, x_{n}\right)-D_{f}\left(q, x_{n+1}\right) .
\end{aligned}
$$

Now, we divide the rest of the proof into two parts as follows.

Case 1. Assume that there exists $n_{0} \in \mathbb{N}$ such that $D_{f}\left(q, x_{n}\right)$ is decreasing for all $n \geq n_{0}$. It then follow that $D_{f}\left(q, x_{n}\right)$ is convergent and hence $D_{f}\left(q, x_{n}\right)-D_{f}\left(q, x_{n+1}\right) \rightarrow 0$ as $n \rightarrow \infty$. Thus, from (3.11) and the conditions on $\alpha_{n}, \beta_{n}, \delta_{n}, \eta_{n}$ and $\theta_{n}$, we get

$$
\lim _{n \rightarrow \infty}\left[\left\|u_{n}-y_{n}\right\|^{2}+\left\|x_{n}-y_{n}\right\|^{2}\right]=\lim _{n \rightarrow \infty}\left[\left\|v_{n}-z_{n}\right\|^{2}+\left\|x_{n}-z_{n}\right\|^{2}\right]=0,
$$


and $\lim _{n \rightarrow \infty} \rho_{r}^{*}\left(\left\|\nabla f x_{n}-T x_{n}\right\|\right)=0$. Hence, $\lim _{n \rightarrow \infty}\left\|u_{n}-y_{n}\right\|=\lim _{n \rightarrow \infty}|| x_{n}-y_{n} \|=0$,

$$
\lim _{n \rightarrow \infty}\left\|v_{n}-z_{n}\right\|=\lim _{n \rightarrow \infty}\left\|x_{n}-z_{n}\right\|=0
$$

and

$$
\lim _{n \rightarrow \infty}\left\|\nabla f x_{n}-T x_{n}\right\|=0
$$

From (3.13) and the fact that $\nabla f^{*}$ is uniformly continuous on bounded subsets of $E^{*}$, we get

$$
\lim _{n \rightarrow \infty}\left\|x_{n}-\nabla f^{*} T x_{n}\right\|=0 \text {. }
$$

Moreover, from (3.9) and Lemma 2.2, we obtain

$$
\begin{aligned}
D_{f}\left(x_{n}, r_{n}\right)= & D_{f}\left(x_{n}, \nabla f^{*}\left[\alpha_{n} \nabla f w+\left(1-\alpha_{n}\right) \nabla f w_{n}\right]\right) \\
\leq & \alpha_{n} D_{f}\left(x_{n}, w\right)+\left(1-\alpha_{n}\right) D_{f}\left(q, w_{n}\right) \\
= & \alpha_{n} D_{f}\left(x_{n}, w\right)+\left(1-\alpha_{n}\right)\left[\beta_{n} D_{f}\left(x_{n}, x_{n}\right)+\delta_{n} D_{f}\left(x_{n}, \nabla f^{*} T x_{n}\right)\right] \\
& +\left(1-\alpha_{n}\right)\left[\eta_{n} D_{f}\left(x_{n}, u_{n}\right)+\theta_{n} D_{f}\left(x_{n}, v_{n}\right)\right] .
\end{aligned}
$$

Thus, from Lemma 2.8, (3.12), (3.14) and (3.15), we get $\lim _{n \rightarrow \infty} D_{f}\left(x_{n}, r_{n}\right)=0$, which implies that $\lim _{n \rightarrow \infty}|| x_{n}-r_{n} \|=0$. Now, since $\left\{x_{n}\right\}$ is bounded in $E$, we have that there exists $u \in E$ and a subsequence $\left\{x_{n_{k}}\right.$ of $\left\{x_{n}\right\}$ such that $x_{n_{k}} \rightarrow u$ and

$$
\limsup _{n \rightarrow \infty}\left\langle x_{n}-q, \nabla f w-\nabla f q\right\rangle=\lim _{k \rightarrow \infty}\left\langle x_{n_{k}}-q, \nabla f w-\nabla f q\right\rangle .
$$

From (3.13) and the fact that $T$ is relatively $f$-nonexpansive, we have $u \in F_{f}(T)$.

Next, we show that $u \in V I\left(C, A_{1}\right) \cap \operatorname{VI}\left(C, A_{2}\right)$. Let

$$
F_{1} x= \begin{cases}A_{1} x+N_{C} x, & x \in C, \\ \emptyset, & x \notin C,\end{cases}
$$

where $N_{C}(x)=\left\{v \in E^{*}:\langle x-z, v\rangle \geq 0, \forall z \in C\right\}$ is the normal cone to $C$. Then, $F_{1}$ is a maximal monotone mapping, and $0 \in F_{1} x$ if and only if $x \in V I\left(C, A_{1}\right)$ (see, e.g., [34]). Let $(x, y) \in$ $G p h\left(F_{1}\right)$. Then $y \in A_{1} x+N_{C}(x)$ and $y-A_{1} x \in N_{C}(x)$. Thus, we obtain

$$
\left\langle x-v, y-A_{1} x\right\rangle \geq 0, \quad \forall v \in C .
$$

Note that $x$ and $u_{n_{k}}$ are in $C$. We have $\left\langle\nabla f x_{n_{k}}-\gamma_{n_{k}} A_{1} y_{n_{k}}-\nabla f u_{n_{k}}, u_{n_{k}}-x\right\rangle \geq 0$, which implies that

$$
\left\langle x-u_{n_{k}}, \frac{\nabla f u_{n_{k}}-\nabla f x_{n_{k}}}{\gamma_{n_{k}}}+A_{1} y_{n_{k}}\right\rangle \geq 0
$$

From (3.17), we find that

$$
\begin{aligned}
\left\langle x-u_{n_{k}}, y\right\rangle \geq & \left\langle x-u_{n_{k}}, A_{1} x\right\rangle \\
\geq & \left\langle x-u_{n_{k}}, A_{1} x\right\rangle-\left\langle x-u_{n_{k}}, \frac{\nabla f u_{n_{k}}-\nabla f x_{n_{k}}}{\gamma_{n_{k}}}+A_{1} y_{n_{k}}\right\rangle \\
= & \left\langle x-u_{n_{k}}, A_{1} x-A_{1} u_{n_{k}}\right\rangle+\left\langle x-u_{n_{k}}, A_{1} u_{n_{k}}-A_{1} y_{n_{k}}\right\rangle \\
& \quad-\frac{1}{\gamma_{n_{k}}}\left\langle x-u_{n_{k}}, \nabla f u_{n_{k}}-\nabla f x_{n_{k}}\right\rangle \\
\geq & \left\langle x-u_{n_{k}}, A_{1} u_{n_{k}}-A_{1} y_{n_{k}}\right\rangle-\frac{1}{\gamma_{n_{k}}}\left\langle x-u_{n_{k}}, \nabla f u_{n_{k}}-\nabla f x_{n_{k}}\right\rangle .
\end{aligned}
$$


Furthermore, from the fact that $A_{1}$ and $\nabla f$ are uniformly continuous, we obtain $\lim _{n \rightarrow \infty} \| A_{1} u_{n}-$ $A_{1} y_{n} \|=0$ and $\lim _{n \rightarrow \infty}\left\|\nabla f u_{n}-\nabla f x_{n}\right\|=0$. Consequently, inequality (3.18) provides that $\langle x-$ $u, y\rangle \geq 0$. Hence, by the maximality of $F_{1}$, we obtain $u \in F_{1}^{-1}(0)$. Therefore, $u \in V I\left(C, A_{1}\right)$. Similarly, we get $u \in V I\left(C, A_{2}\right)$. Thus $u \in V I\left(C, A_{1}\right) \cap V I\left(C, A_{2}\right) \cap F_{f}(T)$. It follows from Lemma 2.4 (i) that

$$
\begin{aligned}
\limsup _{n \rightarrow \infty}\left\langle x_{n}-q, \nabla f w-\nabla f q\right\rangle & =\lim _{k \rightarrow \infty}\left\langle x_{n_{k}}-q, \nabla f w-\nabla f q\right\rangle \\
& =\langle u-q, \nabla f w-\nabla f q\rangle \leq 0 .
\end{aligned}
$$

Therefore, from (3.10), (3.19) and Lemma 2.10, we conclude that $D_{f}\left(q, x_{n}\right) \rightarrow 0$ as $n \rightarrow \infty$. By using Lemma 2.8, we conclude that $x_{n} \rightarrow q$ as $n \rightarrow \infty$.

Case 2. Suppose that there exists a subsequence $\left\{n_{i}\right\}$ of $\{n\}$ such that $D_{f}\left(q, x_{n_{i}}\right)<D_{f}\left(q, x_{n_{i}+1}\right)$, $\forall i \in \mathbb{N}$. It follows from Lemma 2.11 that there exists a nondecreasing sequence $\left\{m_{k}\right\} \subset \mathbb{N}$ such that $m_{k} \rightarrow \infty$ as $k \rightarrow \infty$ and $\max \left\{D_{f}\left(q, x_{m_{k}}\right), D_{f}\left(q, x_{k}\right)\right\} \leq D_{f}\left(q, x_{m_{k}+1}\right)$, for all $k \in \mathbb{N}$. Thus, from (3.11) and the conditions on $\alpha_{n}, \beta_{n}, \delta_{n}, \eta_{n}$ and $\theta_{n}$, we get

$$
\begin{aligned}
& \lim _{k \rightarrow \infty}\left\|u_{m_{k}}-y_{m_{k}}\right\|=\lim _{k \rightarrow \infty}|| x_{m_{k}}-y_{m_{k}} \|=0, \\
& \lim _{k \rightarrow \infty}\left\|v_{m_{k}}-z_{m_{k}}\right\|=\lim _{k \rightarrow \infty}|| x_{m_{k}}-z_{m_{k}} \|=0,
\end{aligned}
$$

and

$$
\lim _{k \rightarrow \infty}\left\|\nabla f x_{m_{k}}-T x_{m_{k}}\right\|=0 .
$$

Moreover, following the methods in Case 1, we get $\lim _{k \rightarrow \infty} \| x_{m_{k}}-r_{m_{k}}||=0$, and

$$
\limsup _{k \rightarrow \infty}\left\langle x_{m_{k}}-q, \nabla f w-\nabla f q\right\rangle \leq 0 .
$$

Now, from (3.10), we obtain

$$
\begin{aligned}
\alpha_{m_{k}}\left(D_{f}\left(q, x_{m_{k}}\right) \leq\right. & D_{f}\left(q, x_{m_{k}}\right)-D_{f}\left(q, x_{m_{k}+1}\right)+\alpha_{m_{k}}|| x_{m_{k}}-r_{m_{k}}|| \mid \nabla f w-\nabla f q \| \\
& +\alpha_{m_{k}}\left\langle x_{m_{k}}-q, \nabla f w-\nabla f q\right\rangle \\
\leq & \alpha_{m_{k}}|| x_{m_{k}}-r_{m_{k}}|||| \nabla f w-\nabla f q \|+\alpha_{m_{k}}\left\langle x_{m_{k}}-q, \nabla f w-\nabla f q\right\rangle .
\end{aligned}
$$

Consequently, since $\alpha_{m_{k}}>0$, we get

$$
D_{f}\left(q, x_{m_{k}}\right) \leq\left\|x_{m_{k}}-r_{m_{k}}||\right\| \nabla f w-\nabla f q \|+\left\langle x_{m_{k}}-q, \nabla f w-\nabla f q\right\rangle .
$$

Therefore, from (3.20) and (3.21), we obtain $D_{f}\left(q, x_{m_{k}}\right) \rightarrow 0$ as $k \rightarrow \infty$. But, we have $D_{f}\left(q, x_{m_{k}+1}\right)$ $\rightarrow 0$ as $k \rightarrow \infty$. Hence, the fact $D_{f}\left(q, x_{k}\right) \leq D_{f}\left(q, x_{m_{k}+1}\right) \operatorname{implies} \lim _{k \rightarrow \infty} D_{f}\left(q, x_{k}\right)=0$. Using Lemma 2.8, we conclude that $x_{k} \rightarrow q$ as $k \rightarrow \infty$.

We remark that the proof of Theorem 3.2 provides the following result for a common point in the $f$-fixed point set of a Bregman relatively $f$-nonexpansive mapping and in the solution set of variational inequality problems with a finite family of Lipschitz monotone mappings in Banach spaces.

Theorem 3.3. Let $f \in \mathscr{F}(E)$ be a strongly coercive, bounded and uniformly Fréchet differentiable Legendre function, which is strongly convex with constant $\mu>0$ on bounded subsets of $E$. Let $A_{i}: C \rightarrow E^{*}$ be a $L_{i}$-Lipschitz monotone mapping with constant $L_{i}$, for $i=$ 
$1,2, \cdots, N$, and let $T: C \rightarrow E^{*}$ be a Bregman relatively $f$-nonexpansive mapping such that $\Omega=\cap_{i=1}^{N} V I\left(C, A_{i}\right) \cap F_{f}(T) \neq \emptyset$. For arbitrary $x_{0}, w \in C$, define an iterative sequence by

$$
\left\{\begin{array}{l}
y_{n, i}=P_{C}^{f} \nabla f^{*}\left(\nabla f x_{n}-\gamma_{n} A_{i} x_{n}\right), \\
u_{n, i}=P_{C}^{f} \nabla f^{*}\left(\nabla f x_{n}-\gamma_{n} A_{i} y_{n, i}\right), \\
w_{n}=\nabla f^{*}\left(\beta_{n} \nabla f x_{n}+\delta_{n} T x_{n}+\sum_{i=1}^{n} \eta_{n, i} \nabla f u_{n, i}\right), \\
x_{n+1}=P_{C}^{f} \nabla f^{*}\left(\alpha_{n} \nabla f w+\left(1-\alpha_{n}\right) \nabla f w_{n}\right),
\end{array}\right.
$$

where $0<\underline{\gamma} \leq \gamma_{n} \leq \bar{\gamma}<\frac{\mu}{L}$, for $L:=\max \left\{L_{i}: i=1,2, \cdots, N\right\}$ and $\left\{\beta_{n}\right\},\left\{\delta_{n}\right\},\left\{\eta_{n, i}\right\} \subset[\varepsilon, 1) \subset$ $(0,1)$ such that $\beta_{n}+\delta_{n}+\sum_{i=1}^{N} \eta_{n, i}=1$, for all $n \geq 0$. Then, $\left\{u_{n}\right\}$ converges strongly to $x^{*}=$ $P_{\Omega}^{f}(w)$.

If, in Theorem 3.2, $C=E$, then the Bregman projection $P_{C}^{f}$ is reduced to the identity mapping on $E$. Hence, $\operatorname{VI}\left(C, A_{1}\right)=A_{1}^{-1}(0)$ and $\operatorname{VI}\left(C, A_{2}\right)=A_{2}^{-1}(0)$. Thus, we obtain the following corollary for a common point in the $f$-fixed point set of Bregman relatively $f$-nonexpansive mappings and the zero point set of Lipschitz monotone mappings in Banach spaces.

Corollary 3.1. Let $f \in \mathscr{F}(E)$ be a strongly coercive, bounded and uniformly Fréchet differentiable Legendre function which is strongly convex with constant $\mu>0$ on bounded subsets of $E$. Let $A_{i}: E \rightarrow E^{*}$ be a $L_{i}$-Lipschitz monotone mapping with constant $L_{i}$, for $i=1,2$ and $T: E \rightarrow E^{*}$ be a Bregman relatively $f$-nonexpansive mapping such that $\Omega=F_{f}(T) \cap A_{1}^{-1}(0) \cap$ $A_{2}^{-1}(0) \neq \emptyset$. For arbitrary $x_{0}, w \in E$, define an iterative sequence by

$$
\left\{\begin{array}{l}
y_{n}=\nabla f^{*}\left(\nabla f x_{n}-\gamma_{n} A_{1} x_{n}\right), \\
z_{n}=\nabla f^{*}\left(\nabla f x_{n}-\gamma_{n} A_{2} x_{n}\right), \\
w_{n}=\nabla f^{*}\left(\beta_{n} \nabla f x_{n}+\delta_{n} T x_{n}+\eta_{n} \nabla f u_{n}+\theta_{n} \nabla f v_{n}\right), \\
x_{n+1}=\nabla f^{*}\left(\alpha_{n} \nabla f w+\left(1-\alpha_{n}\right) \nabla f w_{n}\right),
\end{array}\right.
$$

where $u_{n}=\nabla f^{*}\left(\nabla f x_{n}-\gamma_{n} A_{1} y_{n}\right), v_{n}=\nabla f^{*}\left(\nabla f x_{n}-\gamma_{n} A_{2} z_{n}\right), 0<\gamma \leq \gamma_{n} \leq \bar{\gamma}<\frac{\mu}{L}$, for $L:=$ $\max \left\{L_{1}, L_{2}\right\}$ and $\left\{\beta_{n}\right\},\left\{\delta_{n}\right\},\left\{\eta_{n}\right\},\left\{\theta_{n}\right\} \subset[\varepsilon, 1) \subset(0,1)$ such that $\bar{\beta}_{n}+\delta_{n}+\eta_{n}+\theta_{n}=1$, for all $n \geq 0$. Then, $\left\{x_{n}\right\}$ converges strongly to $x^{*}=P_{\Omega}^{f}(w)$.

If, in Theorem 3.2, $A_{i}=0$, for $i=1,2$, then we obtain a method of approximation for $f$-fixed points of a Bregman relatively $f$-nonexpansive mapping in Banach spaces.

Corollary 3.2. Let $f \in \mathscr{F}(E)$ be a strongly coercive, bounded and uniformly Fréchet differentiable Legendre function, which is strongly convex with constant $\mu>0$ on bounded subsets of $E$. Let $T: C \rightarrow E^{*}$ be a Bregman relatively $f$-nonexpansive mapping such that $F_{f}(T) \neq \emptyset$. For arbitrary $x_{0}, w \in C$, define an iterative sequence by

$$
\left\{\begin{array}{l}
w_{n}=\nabla f^{*}\left(\beta_{n} \nabla f x_{n}+\delta_{n} T x_{n}\right), \\
x_{n+1}=P_{C}^{f} \nabla f^{*}\left(\alpha_{n} \nabla f w+\left(1-\alpha_{n}\right) \nabla f w_{n}\right),
\end{array}\right.
$$

where $\left\{\beta_{n}\right\},\left\{\delta_{n}\right\} \subset[\varepsilon, 1) \subset(0,1)$ such that $\beta_{n}+\delta_{n}=1$, for all $n \geq 0$. Then, $\left\{x_{n}\right\}$ converges strongly to $x^{*}=P_{F_{f}(T)}(w)$.

If, in Theorem 3.3, $C=E$ and $T=\nabla f$, the identity mappong on $E$, then we get the following result for a common zero point of a finite family of Lipschitz monotone mappings in Banach spaces. 
Corollary 3.3. Let $f \in \mathscr{F}(E)$ be a strongly coercive, bounded and uniformly Fréchet differentiable Legendre function, which is strongly convex with constant $\mu>0$ on bounded subsets of $E$. Let $A_{i}: E \rightarrow E^{*}$ be a $L_{i}$-Lipschitz monotone mapping with Lipschitz constant $L_{i}$, for $i=1,2, \cdots, N$ such that $\Omega=\cap_{i=1}^{N} A_{i}^{-1}(0) \neq \emptyset$. For arbitrary $x_{0}, w \in E$, define an iterative sequenc by

$$
\left\{\begin{array}{l}
y_{n, i}=\nabla f^{*}\left(\nabla f x_{n}-\gamma_{n} A_{i} x_{n}\right), \\
u_{n, i}=\nabla f^{*}\left(\nabla f x_{n}-\gamma_{n} A_{i} y_{n, i}\right), \\
w_{n}=\nabla f^{*}\left(\beta_{n, 0} \nabla f x_{n}+\sum_{i=1}^{n} \beta_{n, i} \nabla f u_{n, i}\right), \\
x_{n+1}=\nabla f^{*}\left(\alpha_{n} \nabla f w+\left(1-\alpha_{n}\right) \nabla f w_{n}\right),
\end{array}\right.
$$

where $0<\gamma \leq \gamma_{n} \leq \bar{\gamma}<\frac{\mu}{L}$, for $L:=\max \left\{L_{i}: i=1,2, \cdots, N\right\}$ and $\left\{\beta_{n, i}\right\} \subset[\varepsilon, 1) \subset(0,1)$ such that $\sum_{i=0}^{N} \beta_{n, i}=1$, for all $n \geq 0$. Then, $\left\{x_{n}\right\}$ converges strongly to an element $x^{*}=P_{\Omega}^{f}(w)$.

If, in Corollary 3.1, $E$ is a smooth, strictly convex and real reflexive Banach space, then $f(x)=\frac{1}{2}\|x\|^{2}$ is a strongly coercive, bounded and uniformly Frêchet differentiable Legendre function, which is strongly convex with constant $\mu=2$ and conjugate $f^{*}\left(x^{*}\right)=\frac{1}{2}\left\|x^{*}\right\|^{2}$. This yields that $\nabla f=J_{2}=J$ with $\nabla f^{*}=J_{2}^{-1}=J^{-1}$. Thus, we easily have the following results.

Corollary 3.4. Let $E$ be a smooth, strictly convex and real reflexive Banach space with its dual $E^{*}$ Let $A_{i}: C \rightarrow E^{*}$ be a $L_{i}$-Lipschitz monotone mapping with Lipschitz constant $L_{i}$, for $i=1,2$, and let $T: C \rightarrow E^{*}$ be a relatively $J$-nonexpansive mapping such that $\Omega=F_{f}(T) \cap A_{1}^{-1}(0) \cap$ $A_{2}^{-1}(0) \neq \emptyset$. For arbitrary $x_{0}, w \in C$, define an iterative sequence by

$$
\left\{\begin{array}{l}
y_{n}=\Pi_{C} J^{-1}\left(J x_{n}-\gamma_{n} A_{1} x_{n}\right), \\
z_{n}=\Pi_{C} J^{-1}\left(J x_{n}-\gamma_{n} A_{2} x_{n}\right), \\
w_{n}=J^{-1}\left(\beta_{n} J x_{n}+\delta_{n} T x_{n}+\eta_{n} J u_{n}+\theta_{n} J v_{n}\right), \\
x_{n+1}=\Pi_{C} J^{-1}\left(\alpha_{n} J w+\left(1-\alpha_{n}\right) J w_{n}\right),
\end{array}\right.
$$

where $u_{n}=\Pi_{C} J^{-1}\left(J x_{n}-\gamma_{n} A_{1} y_{n}\right), v_{n}=\Pi_{C} J^{-1}\left(J x_{n}-\gamma_{n} A_{2} z_{n}\right), 0<\gamma \leq \gamma_{n} \leq \bar{\gamma}<\frac{1}{L}$, for $L:=$ $\max \left\{L_{1}, L_{2}\right\}$ and $\left\{\beta_{n}\right\},\left\{\delta_{n}\right\},\left\{\eta_{n}\right\},\left\{\theta_{n}\right\} \subset[\varepsilon, 1) \subset(0,1)$ such that $\overline{\beta_{n}}+\delta_{n}+\eta_{n}+\theta_{n}=1$, for all $n \geq 0$. Then, $\left\{x_{n}\right\}$ converges strongly to $x^{*} \in \Omega$.

Corollary 3.5. Let $E$ be a smooth, strictly convex and real reflexive Banach space with its dual $E^{*}$ Let $A_{i}: C \rightarrow E^{*}$ be a $L_{i}$-Lipschitz monotone mapping with Lipschitz constant $L_{i}$, for $i=1,2$, and let $T: C \rightarrow E$ be a relatively nonexpansive mapping such that $\Omega=\operatorname{VI}\left(C, A_{1}\right) \cap \operatorname{VI}\left(C, A_{2}\right) \cap$ $F(T) \neq \emptyset$. For arbitrary $x_{0}, w \in C$, define an iterative sequence by

$$
\left\{\begin{array}{l}
y_{n}=\Pi_{C} J^{-1}\left(J x_{n}-\gamma_{n} A_{1} x_{n}\right), \\
z_{n}=\Pi_{C} J^{-1}\left(J x_{n}-\gamma_{n} A_{2} x_{n}\right), \\
w_{n}=J^{-1}\left(\beta_{n} J x_{n}+\delta_{n} J T x_{n}+\eta_{n} J u_{n}+\theta_{n} J v_{n}\right), \\
x_{n+1}=\Pi_{C} J^{-1}\left(\alpha_{n} J w+\left(1-\alpha_{n}\right) J w_{n}\right),
\end{array}\right.
$$

where $u_{n}=\Pi_{C} J^{-1}\left(J x_{n}-\gamma_{n} A_{1} y_{n}\right), v_{n}=\Pi_{C} J^{-1}\left(J x_{n}-\gamma_{n} A_{2} z_{n}\right), 0<\gamma \leq \gamma_{n} \leq \bar{\gamma}<\frac{1}{L}$, for $L:=$ $\max \left\{L_{1}, L_{2}\right\}$ and $\left\{\beta_{n}\right\},\left\{\delta_{n}\right\},\left\{\eta_{n}\right\},\left\{\theta_{n}\right\} \subset[\varepsilon, 1) \subset(0,1)$ such that $\overline{\beta_{n}}+\delta_{n}+\eta_{n}+\theta_{n}=1$, for all $n \geq 0$. Then, $\left\{x_{n}\right\}$ converges strongly to $x^{*} \in \Omega$. 
If, in Corollary 3.4, we assume $E=H$, a real Hilbert space, and $f(x)=\frac{1}{2}\|x\|^{2}$, then $\nabla f=$ $J_{2}=I$ and $\nabla f^{*}=J_{2}^{-1}=I$, were $I$ is identity mapping on $H$. Thus, we get the following results in Hilbert spaces.

Corollary 3.6. Let $H$ be a real Hilbert space. Let $A_{i}: C \rightarrow H$ be a $L_{i}$-Lipschitz monotone mapping with Lipschitz constant $L_{i}$, for $i=1,2$, and let $T: C \rightarrow E$ be a quasi-nonexpansive mapping such that $(I-T)$ is demiclosed at 0 and $\Omega=A_{1}^{-1}(0) \cap A_{2}^{-1}(0) \cap F(T) \neq \emptyset$. For arbitrary $x_{0}, w \in C$, define an iterative sequence by

$$
\left\{\begin{array}{l}
y_{n}=P_{C}\left(x_{n}-\gamma_{n} A_{1} x_{n}\right), \\
z_{n}=P_{C}\left(x_{n}-\gamma_{n} A_{2} x_{n}\right), \\
w_{n}=\beta_{n} x_{n}+\delta_{n} T x_{n}+\eta_{n} u_{n}+\theta_{n} v_{n}, \\
x_{n+1}=P_{C}\left(\alpha_{n} w+\left(1-\alpha_{n}\right) w_{n}\right),
\end{array}\right.
$$

where $u_{n}=P_{C}\left(x_{n}-\gamma_{n} A_{1} y_{n}\right), v_{n}=P_{C}\left(x_{n}-\gamma_{n} A_{2} z_{n}\right), 0<\underline{\gamma} \leq \gamma_{n} \leq \bar{\gamma}<\frac{1}{L}$, for $L:=\max \left\{L_{1}, L_{2}\right\}$ and $\left\{\beta_{n}\right\},\left\{\delta_{n}\right\},\left\{\eta_{n}\right\},\left\{\theta_{n}\right\} \subset[\varepsilon, 1) \subset(0,1)$ such that $\beta_{n}+\delta_{n}+\eta_{n}+\theta_{n}=1$, for all $n \geq 0$. Then, $\left\{x_{n}\right\}$ converges strongly to $x^{*} \in \Omega$.

\section{CONCLUSION}

In this paper, we gave the concepts of Bregman relatively $f$-nonexpansive mappings and investigate efficient iterative algorithms for the common solution problem of Bregman relatively $f$-nonexpansive mappings and variational inequality problems with Lipschitz monotone mappings in reflexive real Banach spaces. Theorem 3.2 extends the results in $[2,4,9,12,35]$ from real Hilbert spaces to real reflexive Banach spaces. Corollary 3.5 extends of [13, Theorem 3.1] from 2-uniformly convex and uniformly smooth real Banach spaces to smooth, strictly convex and reflexive real Banach spaces.

\section{Acknowledgements}

The authors gratefully acknowledges the funding from Simons Foundation based at Botswana International University of Science and Technology (BIUST).

\section{REFERENCES}

[1] P. Hartman, G. Stampacchia On some non-linear elliptic differential-functional equations, Acta Math. 115 (1966), 271-310.

[2] N. Nadezhkina, W. Takahashi, Weak convergence theorem by an extragradient method for nonexpansive mappings and monotone mappings, J. Optim. Theory Appl. 128 (2006), 191-201.

[3] D.V. Thong, D. V. Hieu, Weak and strong convergence theorems for variational inequality problems, Numer. Algo. 78 (2018), 1045-1060.

[4] H. Iiduka , W. Takahashi, M. Toyoda M, Approximation of solutions of variational inequalities for monotone mappings, Panam. Math. J. 14 (2004), 49-61.

[5] X. Qin, L. Wang, J.C. Yao, Inertial splitting method for maximal monotone mappings, J. Nonlinear Convex Anal. 21 (2020), 2325-2333.

[6] M. Tian, G. Xu, Inertial modified Tseng's extragradient algorithms for solving monotone variational inequalities and fixed point problems, J. Nonlinear Funct. Anal. 2020 (2020), Article ID 35.

[7] T.M. Tuyen, T.X. Quy, Nguyen Minh Trang, A parallel iterative method for solving a class of variational inequalities in Hilbert spaces, J. Nonlinear Var. Anal. 4 (2020), 357-376. 
[8] S.Y. Cho, S.M. Kang, Approximation of common solutions of variational inequalities via strict pseudocontractions, Acta Math. Sci. 32 (2012), 1607-1618.

[9] W. Takahashi, M. Toyoda, Weak convergence theorems for nonexpansive mappings and monotone mappings, J. Optim. Theory Appl. 118 (2003), 417-428.

[10] L.C. Ceng, Asymptotic inertial subgradient extragradient approach for pseudomonotone variational inequalities with fixed point constraints of asymptotically nonexpansive mappings, Commun. Optim. Theory 2020 (2020), Article ID 2.

[11] L. Liu, A hybrid steepest descent method for solving split feasibility problems involving nonexpansive mappings, J. Nonlinear Convex Anal. 20 (2019), 471-488.

[12] Y. Zhanga, Q. Yuan, Iterative common solutions of fixed point and variational inequality problems, J. Nonlinear Sci. Appl. 9 (2016), 1882-1890.

[13] A.R. Tufa, H. Zegeye, An algorithm for finding a common point of the solutions of fixed point and variational inequality problems in Banach spaces, Arab. J. Math. 4 (2015), 199-213.

[14] R.P. Phelps, Convex Functions, Monotone Operators, and Differentiability, Lecture Notes in Mathematics, vol. 1364, 2nd edn. Springer Verlag, Berlin, 1993.

[15] H. H. Bauschke, J. M. Borwein, Legendre functions and the method of random Bregman projections, J. Convex Anal. 4 (1997), 27-67.

[16] H. H. Bauschke, J. M. Borwein, P.L. Combettes, Essential smoothness, essential strict convexity, and Legendre functions in Banach spaces, Comm. Contemp. Math. 3 (2001), 615-647.

[17] Y. Censor, A. Lent, An iterative row-action method for interval convex programming, J. Optim. Theory Appl. 34 (1981), 321-353.

[18] S. Reich, S. Sabach, Two strong convergence theorems for Bregman strongly nonexpansive operators in re exive Banach spaces, Nonlinear Anal. 73 (2010), 122-135.

[19] S. Reich, S. Sabach, A projection method for solving nonlinear problems in reflexive Bnanch spaces, J. Fixed Point Theory Appl. 9 (2011), 101-116.

[20] D. Butnariu, A.N. Iusem, Totally Convex Functions for Fixed Points Computation and Infinite Dimentional Optimization, Vol. 40, Klumer Academic, Dodrecht, The Netherlands, 2000.

[21] C. Zalinescu, Convex Analysis in General Vector Spaces, World Scientific, River Edge, NJ, 2002.

[22] S. Reich, S. Sabach, A strong convergence theorem for a proximal-type algorithm in reflexive Banach spaces, J. Nonlinear Convex Anal. 10 (2009), 471-485.

[23] D. Butnariu, E. Resmerita, Bregman distances, totally convex functions and a method for solving operator equations in Banach spaces, Abstr. Appl. Anal. 2006 (2006), 1-39.

[24] J. F. Bonnans, A. Shapiro, Perturbation Analysis of Optimization Problem, Springer, New York, 2000.

[25] P. Senakka, P. Cholamjiak, Approximation method for solving fixed point problem of Bregman strongly nonexpansive mappings in reflexive Banach spaces, Ric. Mat. 65 (2016), 209-220.

[26] S. Matsushita, W. Takahashi, Weak and strong convergence theorems for relatively nonexpansive mappings in Banach spaces, Fixed Point Theory Appl. 2004 (2004), 37-47.

[27] C. E. Chidume, C.G. Chinedu, New algorithms for approximating zeros of inverse strongly monotone maps and $J$-fixed points, Fixed Point Theory Appl. 2020 (2020), 3.

[28] D. Bertsekas, Convex Analysis and Optimization Contributors, Angelia Nedic and Asuman E. Ozdaglar. Athena Scientific, 2003,

[29] Y. Nesterov Introductory Lectures on Convex Optimization A Basic Course, Kluwer Academic Publishers, 2004 ,

[30] E. Naraghirad, C.J. Yao, Bregman weak relatively nonexpanseve mappings in Banach spaces, Fixed point Theory Appl. 2013 (2013), 141.

[31] G.B. Wega, H. Zegeye, Convergence results of Forward-Backward method for a zero of the sum of maximally monotone mappings in Banach spaces. Comput. Appl. Math. 39 (2020), 223.

[32] H.-K. Xu, Viscosity approximation methods for nonexpansive mappings, J. Math. Anal. Appl. 298 (2004), 279-291.

[33] P.E. Maingé, Strong convergence of projected subgradiant method for nonsmooth and nonstrictily convex minimization, Set-Valued Anal. 16 (2008), 899-912. 
[34] R.T. Rockafellar, On the maximality of sums of nonlinear monotone operators, Trans. Am. Math. Soc. 149 (1970), 75-88.

[35] A. Tada, W. Takahashi, Weak and strong convergence theorems for nonexpansive mappings and equilbrium problems, J. Optim. Theory Appl. 133 (2007), 359-370. 